\title{
Numerical Studies of the Solar Energetic Particle Transport and Acceleration
}

\author{
V. Tenishev ${ }^{*}$, M. Combi ${ }^{\dagger}$, I. Sokolov ${ }^{\ddagger}$ I. Roussev ${ }^{\S}$, T. Gombosi ${ }^{\sharp}$ \\ Space Physics Research Laboratory, Department of Atmospheric, Oceanic and Space Sciences, University of \\ Michigan, Ann Arbor, MI 48109
}

\begin{abstract}
Solar energetic particles often get accelerated to energies up to few $\mathrm{GeV}$ at interplanetary shock waves driven by Coronal Mass Ejections (CMEs) and are of considerable importance for space weather studies because they can produce radiation hazards for manned or unmanned spacecraft. Particles accelerated at the shock wave can escape upstream and downstream into the interplanetary medium. As the escaped high-energy particles propagate along the interplanetary magnetic field, they are scattered by fluctuations of the turbulent magnetic field.

The Monte-Carlo method has been adopted in this work to study propagation and scattering of solar energetic particles. We have demonstrated that high energy particles reach the orbit of the Earth before the bulk flow, which due to its high intensity produces main hazard. The detection of high energy particles may serve as a precursor of its arrival.
\end{abstract}

\section{Introduction}

The solar energetic particle (SEP) events associated with coronal mass ejections (CMEs) are of particular importance for space weather studies. High energy solar protons $(\sim$ few $\mathrm{GeV})$ can be accelerated within a short period of time ( $\leq 1 \mathrm{hr}$ ) after the initiation of solar eruptions, which makes them difficult to predict, and pose a serious concern for the design and operation of both manned and unmanned space missions. Recent theories and related observations ${ }^{1-7}$ suggest that these high-energy particles are the result of the first-order Fermi acceleration process ${ }^{8}$ at a shock wave driven by a solar eruption, the so-called diffusive shock acceleration (DSA), in the Sun's proximity $\left(2-15 R_{\odot}\right)$. These theories, however, have been debated within the community ${ }^{9-11}$ because very little is known about the dynamical properties of CME driven shock waves in the inner corona soon after the onset of the eruption and whether or not the level of turbulence at the shock is sufficient for this mechanism to work.

\section{Energetic Particle Transport and Acceleration}

While diffusing through highly turbulent media in the vicinity of the front of a CME-driven shock wave, solar energetic particles suffer scattering due to turbulent fluctuations of the magnetic field. The evolution of the distribution function, $f(\mathbf{x}, \mathbf{p}, t)$, of energetic particles can be described ${ }^{12}$ by the cosmic ray transport equation

$$
\frac{\partial f}{\partial t}=\nabla \cdot(\mathbf{k} \cdot \nabla f)-(\mathbf{U} \cdot \nabla) f+\frac{1}{3}(\nabla \cdot \mathbf{U}) \frac{\partial f}{\partial \ln p}+Q,
$$

where $\mathbf{U}$ is the plasma velocity, $p=|\mathbf{p}|$ is the particle momentum, $\mathbf{k}$ is the diffusion tensor that is determined by the spectrum of magnetic fluctuations and $Q(\mathbf{x}, \mathbf{p}, t)$ is the injection rate. Equation (1) is a good approximation ${ }^{13}$

\footnotetext{
${ }^{*}$ Research Assistant, Department of Atmospheric, Oceanic and Space Sciences

${ }^{\dagger}$ Research Professor, Department of Atmospheric, Oceanic and Space Sciences

* Associate Research Scientist, Department of Atmospheric, Oceanic and Space Sciences

$\S$ Assistant Research Scientist, Department of Atmospheric, Oceanic and Space Sciences

\# Professor, Department of Atmospheric, Oceanic and Space Sciences
} 
for energetic $\left(p \gg m_{p}|\mathbf{U}|\right)$ particles and results in a power law spectrum of isotropic part of canonical distribution function of energetic particles, $f_{s w}(p)$, at the front of the shock wave

$$
\begin{gathered}
f_{s w}(p) \sim p^{-q}, p \in\left(p_{\text {inj }}, p_{\max }\right), \\
q=\frac{3 s}{s-1} .
\end{gathered}
$$

Here, $p_{\text {inj }}$ and $p_{\max }$ are particle injection and maximum momenta, $q$ is the spectral index and $s$ is the compression ratio of the shock wave. The fraction, $\eta_{i n j}$, of the post shock solar wind with density $n_{2}$ that are injected into the cosmic rays depends ${ }^{14}$ on the injection parameter $C_{1}$

$$
\eta_{i n j} \equiv \frac{n_{i n j}}{n_{2}}=8 \sqrt{\frac{2}{\pi}} c_{1}^{3} e^{-2 c_{1}^{2}} \frac{\left(p_{\max } / p_{\text {inj }}\right)^{3-q}-1}{3-q},
$$

where $n_{i n j}$ is the number density of injected particles. To agree with the particle fluxes observed at $1 \mathrm{AU}, \eta_{i n j}$ should be ranging between $10^{-4}$ and few $\times 10^{-3}$.

Energetic particles escaping the front of the shock wave obey the Boltzmann equation

$$
\begin{gathered}
\frac{\partial f}{\partial t}+\frac{\mathbf{p}}{m_{p}} \cdot \nabla f+\mathbf{F} \cdot \nabla_{p} f=\left(\frac{\delta f}{\delta t}\right)_{\text {coll }}, \\
\mathbf{F}=\frac{\mathbf{p}}{m_{p}} \times \mathbf{B} .
\end{gathered}
$$

Here, $\mathbf{B}$ is the mean interplanetary magnetic field approximated by the Parker spiral

$$
\mathbf{B}=B_{0}\left(\frac{R_{0}}{r}\right)^{2} \mathbf{e}_{r}-B_{0}\left(\frac{R_{0}}{r}\right)^{2}\left(r-R_{0}\right) \frac{\Omega_{\odot} \sin \theta}{u_{s w}} \mathbf{e}_{\phi},
$$

where $\theta$ is the co-latitude with respect to the solar rotation axis, $B_{0}$ is the interplanetary magnetic field at the corotation radius $R_{0}\left(R_{0}=10 R_{\odot}, B_{0}=1.83 \times 10^{-6} T, u_{s w}=400 \mathrm{~km} / \mathrm{s}, \Omega_{\odot}=2 \pi / 25.4\right.$ days $)$. In this work, the equatorial plane, where $\theta=\pi / 2$, has been considered.

For charged particles propagating through the solar wind, the collision integral, $(\delta f / \delta t)_{\text {coll }}$, is due to scattering from fluctuations of the interplanetary magnetic field, which can be described in terms of a mean free path, $\lambda_{\|}$, that has been adopted ${ }^{4}$ in the form

$$
\frac{\lambda_{\|}}{10^{6} \mathrm{~km}}=8.30 \frac{\left(B / B_{0}\right)^{2}}{\delta B_{x}^{2} / \delta B_{x_{0}}^{2}}\left(\frac{l}{l_{0}}\right)^{2 / 3}\left(\frac{p / M_{n} c}{B / B_{0}}\right)^{1 / 3}(1+C),
$$

where $p$ is the particle momentum, $M_{n} c^{2}=938 \mathrm{MeV}$ is the rest energy of protons, $l_{0}=3.4 \times 10^{6} \mathrm{~km}$ is the turbulent correlation length, $B_{0}=4.12 \mathrm{nT}$ and $\delta B_{x_{0}}^{2}=13.2152(\mathrm{nT})^{2}$ are parameters with typical values evaluated at a heliocentric distance of $1 \mathrm{AU}$ and $C$ is a correction factor. For the purpose of practical simulation, expression (8) has been reformulated ${ }^{4}$ in the form

$$
\lambda_{\|}=\lambda_{0}\left(\frac{p c}{1 \mathrm{GeV}}\right)^{\alpha}\left(\frac{r}{1 \mathrm{AU}}\right)^{\beta} \text {. }
$$


Here, $\lambda_{0}$ is an empirical parameter. The parameters $\alpha$ and $\beta$ describe the momentum and the heliocentric dependence of the mean free path, respectively. Correspondingly, the probability density, $p(h)$, for the distance between two consecutive scattering events, $h$, is taken in the form

$$
p(h)=e^{-h / \lambda_{\|}} .
$$

Between two consecutive pitch angle scatterings, charged particles move through the solar wind plasma gyrating about the magnetic field lines (7).

\section{Monte-Carlo Approach}

The numerical procedure described below has been developed on the basis on the test particle Monte-Carlo method. A test particle can be characterized by its radius vector $\mathbf{r}$ and momentum $\mathbf{p}$. So, the distribution function of solar energetic particles is approximated in the form

$$
f(\mathbf{r}, \mathbf{p})=\sum_{i} w_{i} \delta\left(\mathbf{r}-\mathbf{r}_{i}\right) \delta\left(\mathbf{p}-\mathbf{p}_{i}\right),
$$

where $w_{i}$ is a statistical weight of the particle, i.e. the number of real particles represented by a model one. The evolution of the distribution function (11) is modeled by tracing trajectories of individual model particles, the time of injection, $t_{i}$, and initial momentum, $\mathbf{p}_{i}$, being sampled as described below.

Denoting the position of the shock front as $R_{s h}$ and the velocity of its front as $V_{s h}$, the number of real particles leaving the shock wave within the time interval $d t$ is

$$
d N=Q(t) V_{s h} d s d t
$$

where $Q(t) \sim r^{-2}$ is the injection rate per unit area and $d s \sim 1 / B_{r} \sim r^{2}$ is the cross section area are of magnetic tube. The time of injection, $t_{i}$, can be obtained by solving

$$
\int_{0}^{t_{i}} V_{s h}(t) d t^{\prime}=\xi_{1} \int_{0}^{t_{e}} V_{s h}(t) d t^{\prime}
$$

Here, $t_{e}$ is the time instant at which the shock wave reaches the orbit of the Earth and $\xi_{1,2} \in(0,1)$ are random numbers. Once the time of injection is determined, the momentum of a real particle should be sampled according to

$$
\int_{p_{\text {inj }}}^{p_{i}} f_{s w}\left(r\left(t_{i}\right), p, t_{i}\right) p^{2} d p=\xi_{2} \int_{p_{\text {inj }}}^{p_{\max }} f_{s w}\left(r\left(t_{i}\right), p, t_{i}\right) p^{2} d p,
$$

so that the probability for a real particle to have a momentum within the interval of $(p, p+d p)$ is

$$
\left(\frac{d \xi_{2}}{d p}\right)_{\text {real }}=\frac{f_{s w}(r, p, t) p^{2}}{\int_{p_{\text {inj }}}^{p_{\max }} f_{s w}\left(r, p^{\prime}, t\right) p^{\prime 2} d p^{\prime}}
$$

For a model particle this probability $w$ time is less

$$
\left(\frac{d \xi_{2}}{d p}\right)_{\text {model }}=\frac{f_{s w}(r, p, t) p^{2} / w(p)}{\int_{p_{\text {inj }}}^{p_{\max }} f_{s w}\left(r, p^{\prime}, t\right) p^{\prime 2} / w\left(p^{\prime}\right) d p^{\prime}}
$$

One can use $w=$ const, resulting in the choice of 


$$
\left(\frac{d \xi_{2}}{d p}\right)_{\text {real }}=\left(\frac{d \xi_{2}}{d p}\right)_{\text {model }}
$$

However this approach is not efficient for numerical simulation, resulting in a higher level of noise for the highenergy part of the distribution function. In order to have model particles uniformly distributed over the interval of $\ln E \in\left(\ln E\left(p_{i n j}\right), \ln E\left(p_{\max }\right)\right)$, so that

$$
\left(\frac{d \xi_{2}}{d p}\right)_{\text {model }} \cdot \frac{d p(\ln E)}{d(\ln E)}=\text { const },
$$

we chose the weight function $w(\ln E) \sim f_{s w}(r, p, t) p^{2} /\left(d \xi_{2} / d p\right)_{\text {model }}$ :

$$
w=\frac{f_{s w}(r, p(\ln E), t) p^{2}(\ln E)}{\left(\frac{d \xi_{2}}{d p}\right)_{\text {model }}}=\frac{f_{s w}(r, p(\ln E), t) p^{2}(\ln E)}{c o n s t / \frac{d p(\ln E)}{d(\ln E)}}
$$

The final form of the weight function can be written in the form

$$
w=f_{s w}(r, p(\ln E), t) p^{2}(\ln E) \frac{d p(\ln E)}{d(\ln E)} .
$$

The particles that escape the shock wave can experience isotropic scattering with the mean free path (9). It is assumed that between two consecutive scattering events charged particles move along the magnetic field (7). The particle trajectory is traced until either the particle is recaptured by the shock wave or the shock front reaches the heliocentric distance of $1 \mathrm{AU}$.

\section{Results and Discussions}

Two cases that correspond to an idealized strong shock wave with the compression ratio of $s=4$ and a model of a CME-driven shock wave have been considered.

\section{A. Strong shock wave}

The velocity profile shown in Figure IV.1 has been taken from [4]. The study of influence of the heliocentric distance and momentum dependence of the mean free path on the energetic particle spectrum has been carried out by varying parameters $\alpha$ and $\beta$ in the expression (9). The simulation results are presented in Figure IV.2 ( $\alpha=0, \beta=0$ ), Figure IV.3 $(\alpha=1 / 3, \beta=0)$ and Figure IV.4 $(\alpha=0, \beta=2 / 3)$. The characteristic length of the mean free path $\lambda_{0}=0.4 \mathrm{AU}$ has been used for all three cases.

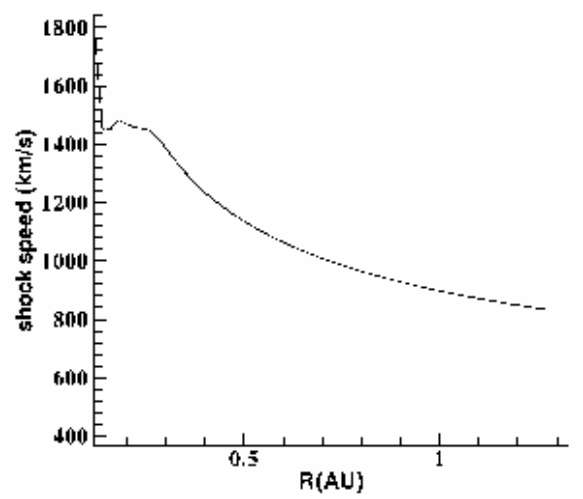

Figure IV.1 Velocity profile of a strong shock wave 

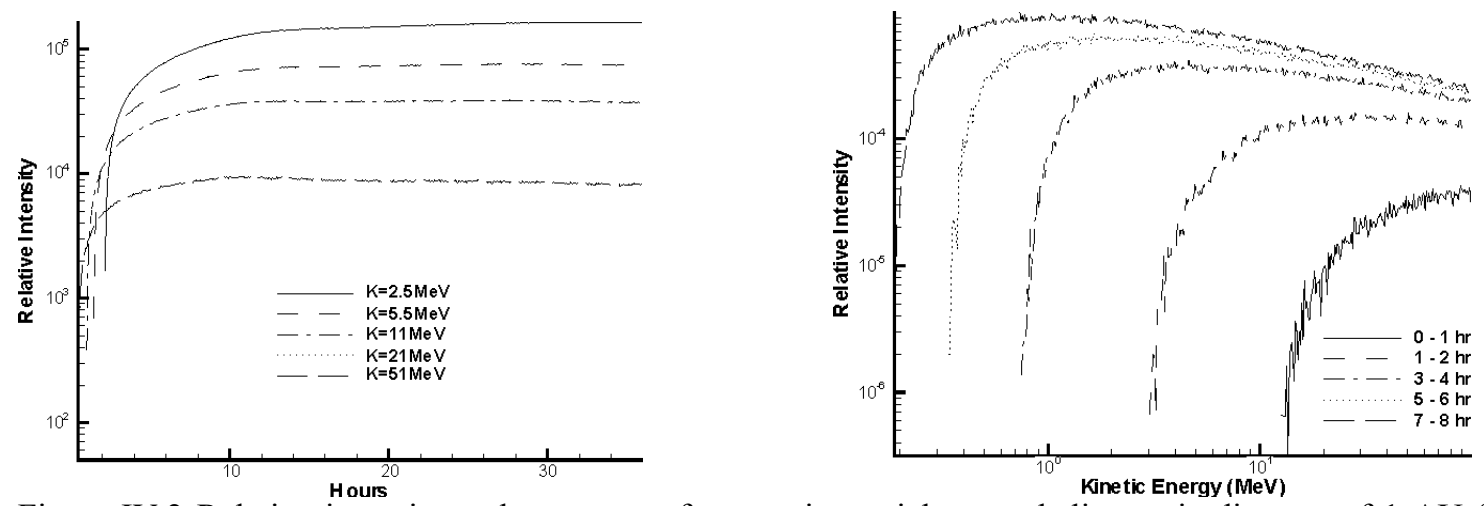

Figure IV.2 Relative intensity and spectrum of energetic particles at a heliocentric distance of $1 \mathrm{AU}$ for a strong shock wave and mean free path (9) parameters $\alpha=0, \beta=0, \lambda_{0}=0.4 \mathrm{AU}$
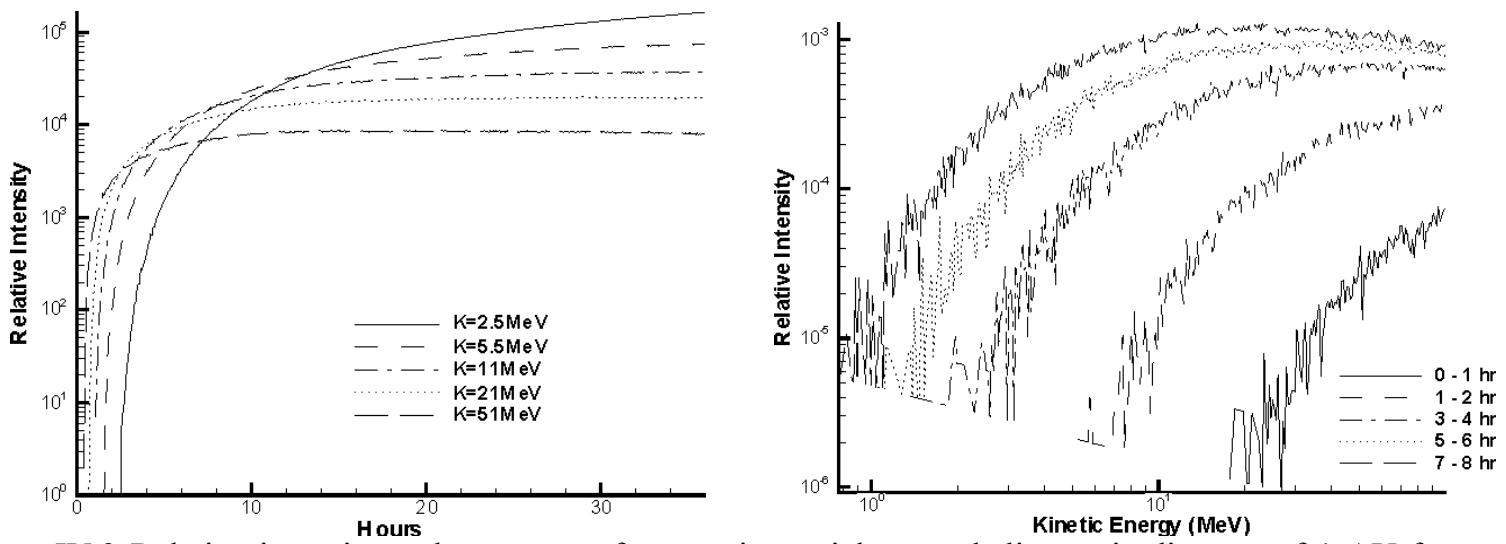

Figure IV.3 Relative intensity and spectrum of energetic particles at a heliocentric distance of $1 \mathrm{AU}$ for a strong shock wave and mean free path (9) parameters $\alpha=1 / 3, \beta=0, \lambda_{0}=0.4 \mathrm{AU}$
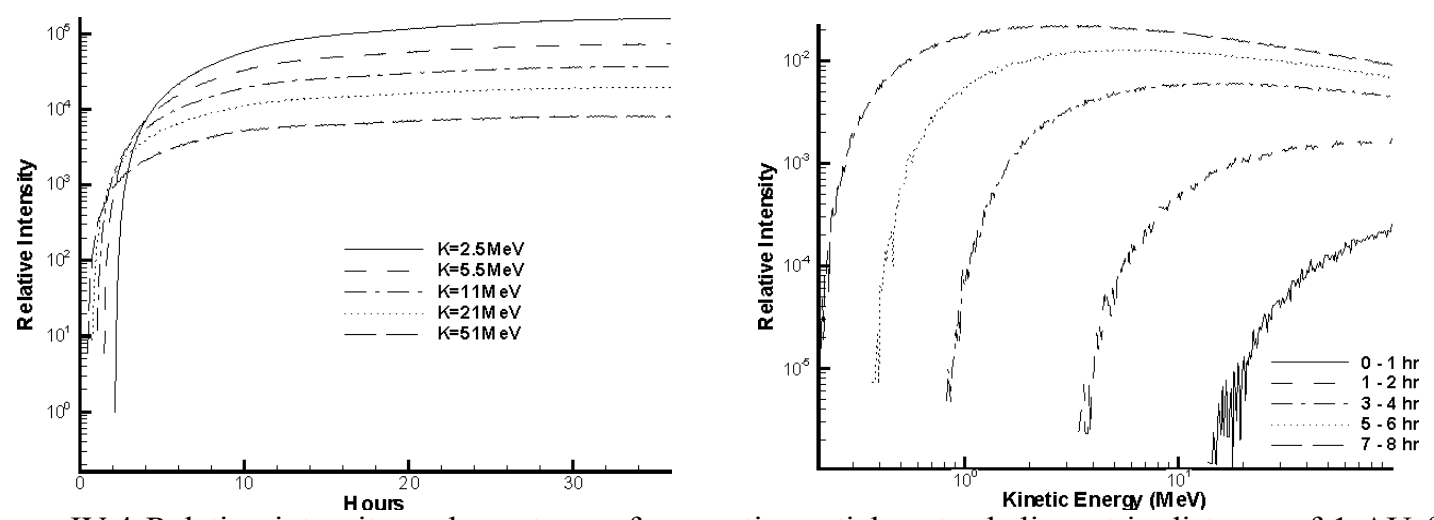

Figure IV.4 Relative intensity and spectrum of energetic particles at a heliocentric distance of $1 \mathrm{AU}$ for a strong shock wave and mean free path (9) parameters $\alpha=0, \beta=2 / 3, \lambda_{0}=0.4 \mathrm{AU}$ 


\section{B. CME-driven shock wave}

The parameters of the shock wave that have been used in this example are the result of a numerical simulation of a shock wave propagation that corresponds to the May 2, 1998 event $^{11}$. The speed and compression ratio of the wave are given in Figure IV.5. The spectrum and intensity profiles obtained are given in Figure IV.6 and Figure IV.7. They indicate that high energy solar particles $(\mathrm{K}>51 \mathrm{MeV})$ can reach the orbit of the Earth in a short period of time $(<1 \mathrm{hr})$ after initiation of solar eruption.
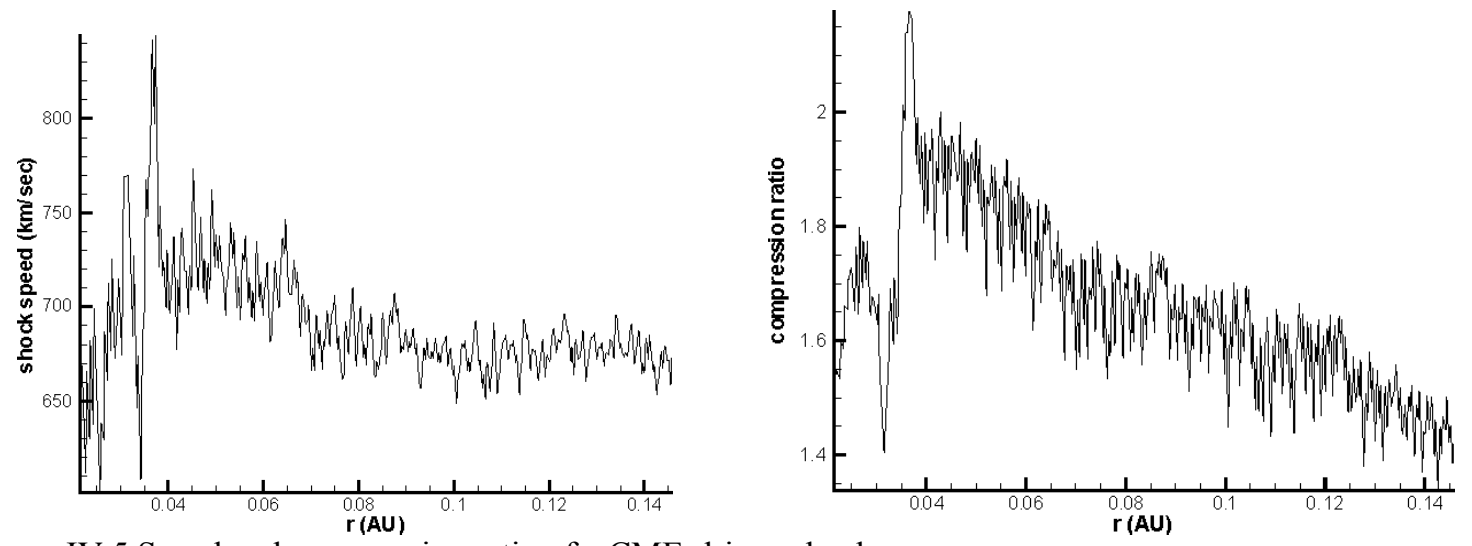

Figure IV.5 Speed and compression ratio of a CME-driven shock wave
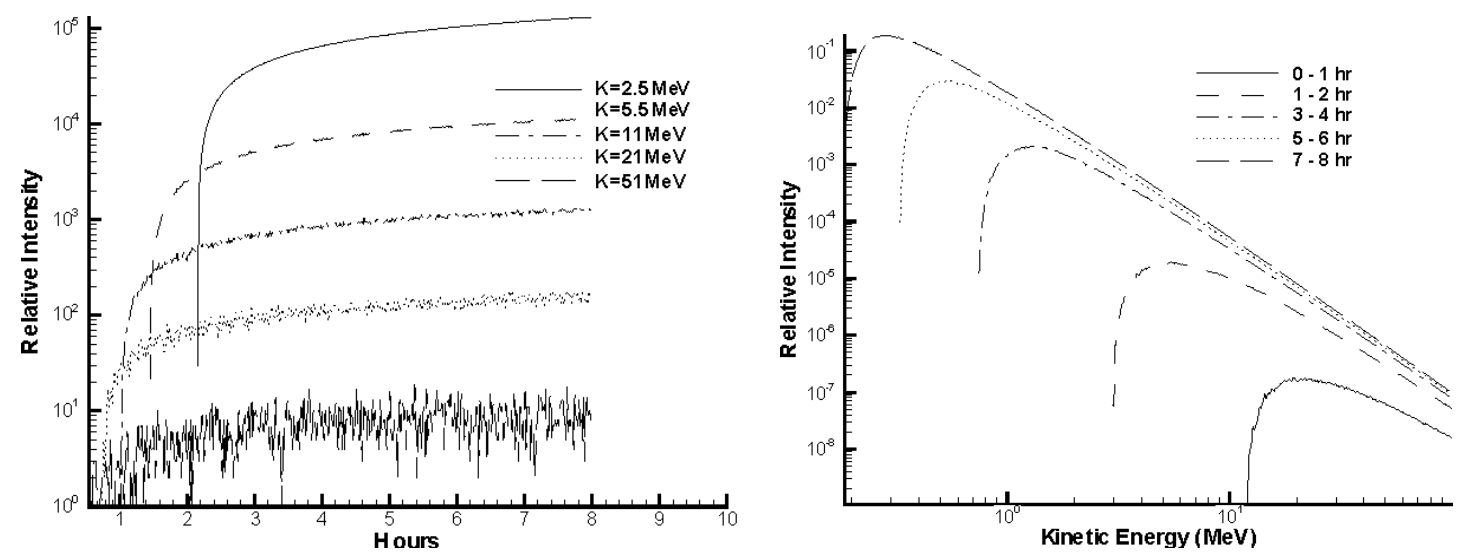

Figure IV.6 Relative intensity and spectrum of energetic particles at a heliocentric distance of 1 AU for a CMEdriven shock wave and mean free path (9) parameters $\alpha=0, \beta=0, \lambda_{0}=0.4 \mathrm{AU}$
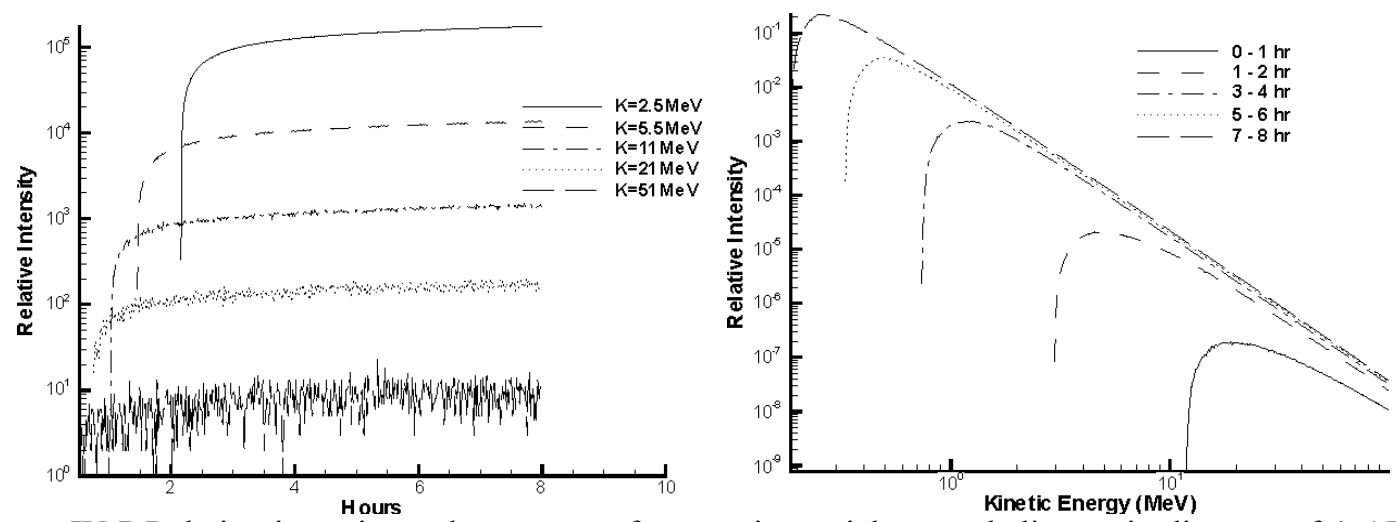

Figure IV.7 Relative intensity and spectrum of energetic particles at a heliocentric distance of 1 AU for a CMEdriven shock wave and mean free path (9) parameters $\alpha=0, \beta=0, \lambda_{0}=1.6 \mathrm{AU}$ 


\section{References}

${ }^{1}$ Cliver, E.W. , Kahler, S.W.; Reames, D.V. Coronal shocks and solar energetic proton events, Astrophysical Journal, v 605 , n 2, pt.1, 20 April 2004, p 902-10

${ }^{2}$ Kahler, S. Injection profiles of solar energetic particles as functions of coronal mass ejection heights, Astrophysical Journal, v 428, n 2, pt.1, 20 June 1994, p 837-42

${ }^{3}$ Lee, M.A. Coupled hydromagnetic wave excitation and ion acceleration at interplanetary traveling shocks, Journal of Geophysical Research, v 88, n A8, 1 Aug. 1983, p 6109-19 4

${ }^{4}$ Gang Li, Zank, G.P., Rice, W.K.M., Energetic particle acceleration and transport at coronal mass ejection-driven shocks,Journal of Geophysical Research, v 108, n A2, 1 Feb. 2003, p SSH10-1-22

${ }^{5} \mathrm{Ng}$, C.K., Reames, D.V., Tylka, A.J., Effect of proton-amplified waves on the evolution of solar energetic particle composition in gradual events, Geophysical Research Letters, v 26, n 14, 15 July 1999, p 2145-8

${ }^{6} \mathrm{Ng}$, C.K., Reames, D.V., Tylka, A.J., Modeling shock-accelerated solar energetic particles coupled to interplanetary Alfven waves, Astrophysical Journal, v 591, n 1, pt.1, 1 July 2003, p a461-85

${ }^{7}$ Zank, G.P., Rice, W.K.M., Wu, C.C., Particle acceleration and coronal mass ejection driven shocks: a theoretical model,Journal of Geophysical Research, v 105, n A11, 1 Nov. 2000, p 25079-95

${ }^{8}$ Fermi, E., On the origin of the cosmic radiation, Physical Review, v 75, 15 April 1949, p 1169-1174

${ }^{9}$ Bulanov, S.V., Sokolov, I.V., The effectiveness of charged particles acceleration by shock waves in supernova remnants and in solar flares, Soviet Astron. Lett., 10, 247, 1984

${ }^{10}$ Reames, D.V.,Particle acceleration at the Sun and in the heliosphere,Space Science Reviews, v 90, n 3-4, 1999, p 413-91

${ }^{11}$ Sokolov, I. V., Roussev, I. I., Gombosi, T. I., Lee, M. A., Kota, J., Forbes, T. G., Manchester, W. B., \& Sakai, J. I, "New Field-Line-Advection Model for Solar Particle Acceleration",Astrophysical Journal Letters, 616: L171-174, 2004

${ }^{12}$ Parker E., The passage of energetic charged particles through interplanetary space, Planet Space Science, 13, 1965

${ }^{13}$ Jokipii, J. R., Acceleration and transport of energetic charged particles in space, Astrophysics and Space Science, 277, 1526,2001

${ }^{14}$ Miniati, F., Ryu D., Kang H., Jones T., Cosmic Ray protons accelerated at cosmological shocks and their impact on groups and clusters of galaxies, The Astrophysical Journal, 559:59-69, 2001 